\title{
Оценка точности вычисления показателей
} гемодинамики и массы миокарда левого желудочка по данным ЭКГ-синхронизированной перфузионной сцинтиграфии миокарда: сравнение с многосрезовой компьютерной томографией сердца

\section{Саушкин В.В. *, Завадовский К.В.}

Научно-исследовательский институт кардиологии ФГБНУ «Томский национальный исследовательский медицинский центр Российской академии наук», ул. Киевская, 111a, Томск, 634012, Российская Федерация

\begin{abstract}
Резюме
Цель исследования - оценить точность вычисления значений конечного диастолического (КД0), конечного систолического (КСО) объемов, фракции выброса (ФВ) и массы миокарда левого желудочка (ЛЖ), полученных по данным ЭКГ-синхронизированной перфузионной сцинтиграфии миокарда (ЭКГПСМ), на СZТ-камере, в сравнении с многосрезовой компьютерной томографией (МСКТ) сердца.
\end{abstract}

Материал и методы. Были обследованы 34 пациента (средний возраст $62 \pm 5$ лет) с подозрением на ишемическую болезнь сердца (ИБС) или ранее установленным диагнозом ИБС. Всем пациентам была выполнена МСКТ-ангиография коронарных артерий и перфузионная сцинтиграфия миокарда c ${ }^{99 m}$ Тс-МИБИ. Для сравнительного анализа были использованы значения КД0, КСО, ФВ и массы миокарда ЛЖ, определенные по данным ЭКГ-ПСМ в покое и МСКТ сердца. Исследования были проведены на гибридном 64-срезовом 0ЭКТ/КТ-томографе Discovery 570c (GE Healthcare, США).

Результаты. При анализе результатов обоих методов были выявлены статистически значимые различия в значениях КДО (МСКТ: 168 (145-210) мл, ЭКГ-ПСМ: 112 (94-141) мл; p<0,05), КСО (МСКТ: 72 (49-83) мл, ЭКГ-ПСМ: 44 (32-66) мл; p<0,05) и массы ЛЖ (МСКТ: 123 (107-143) г, ЭКГ-ПСМ: 140 (124-168) г; $p<0,05)$. Значения ФВ левого желудочка (МСКТ: 64 (54-69)\%, ЭКГ-ПСМ: 61 (50-66)\%; $p>0,05)$ значимо не различались. Была выявлена статистически значимая корреляция значений КДО, КСО и массы миокарда ЛЖ $(r=0,81, r=0,78, r=0,82$ соответственно; $p<0,05)$ и средняя корреляция ФВ лЖ $(r=0,66$, $p<0,05)$. При проведении анализа Бланда-Альтмана значения КД0, КСО и массы миокарда ЛЖ имели статистически значимые различия. Границы согласованности для показателей были следующими: Кд0 9-105 мл, КС0 9-55 мл, масса миокарда ЛЖ 51,6-20,7 г. Согласованность в измерении была выявлена только для ФВ (границы согласия 16,9-18,4\%, $<<0,05)$. Были вычислены уравнения линейной регрессии, позволяющие получить точные значения объемных показателей и массы миокарда ЛЖ по данным ЭКГ-ПСМ.

Заключение. Сцинтиграфический метод по сравнению с МСКТ показывает значимо меньшие объемы (КДО и КСО) и большие значения массы миокарда левого желудочка. Значения КДО, КСО, ФВ и массы миокарда ЛЖ, вычисленные при помощи ЭКГ-ПСМ, имеют статистически значимую сильную корреляцию с результатами МСКТ. При этом хорошая согласованность результатов измерения была выявлена только для фракции выброса ЛЖ.

Ключевые слова: перфузионная сцинтиграфия миокарда; многосрезовая компьютерная томография; кадмий-цинк-теллур; объемные показатели левого желудочка; масса миокарда левого желудочка.

Конфликт интересов. Авторы заявляют об отсутствии конфликта интересов.

Финансирование. Исследование выполнено в рамках государственного задания № AAAA-A15115123110026-3.

Для цитирования: Саушкин В.В., Завадовский К.В. Оценка точности вычисления показателей гемодинамики и массы миокарда левого желудочка по данным ЭКГ-синхронизированной перфузионной сцинтиграфии миокарда: сравнение с многосрезовой компьютерной томографией сердца. Вестник рентгенологии и радиологии. 2019; 100 (3): 152-60. https://doi.org/10.20862/0042-4676-2019-100-3152-160

Статья поступила 13.03.2018 Принята к печати 26.03.2018

152 Вестник рентгенологии и радиологии | Journal of Radiology and Nuclear Medicine | 2019 | Том 100 | №3 | 152-160 


\title{
Assessment of Accuracy in Calculating Hemodynamic Parameters and Left Ventricular Mass According to ECG-Synchronized Myocardial Perfusion Scintigraphic Data: Comparison with Cardiac Multislice Computed Tomography
}

\author{
Viktor V. Saushkin*, Konstantin V. Zavadovskiy \\ Cardiology Research Institute, Tomsk National Research Medical Center of the Russian Academy of Sciences, \\ ul. Kievskaya, 111a, Tomsk, 634012, Russian Federation
}

\begin{abstract}
Objective. To assess accuracy in calculating the values of end-diastolic and end-systolic volumes (EDV and ESV), ejection fraction (EF), and left ventricular (LV) mass, which are obtained according to ECG-synchronized myocardial perfusion scintigraphy (ECG-MPS) on a CZT camera versus those of cardiac multislice computed tomography (MSCT).

Material and methods. Thirty-four patients (mean age, $62 \pm 5$ years) with suspected coronary heart disease or its previously established diagnosis were examined. All the patients underwent MSCT coronary angiogra-

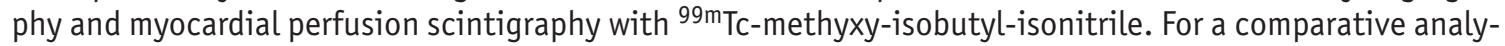
sis, the investigators used the EDV, ESV, FV and LV mass values determined by ECG-MPS at rest and cardiac MSCT. The studies were conducted on a 64-slice SPECT/CT hybrid scanner (Discovery 570c, GE Healthcare, USA).
\end{abstract}

Results. The analysis of the results obtained by both methods revealed statistically significant differences in the values of EDV (MSCT: 168 (145-210) ml; ECG-MPS: 112 (94-141) ml; $p<0.05)$, ESV (MSCT: 72 (49-83) ml; ECG-MPS: 44 (32-66) ml; $p<0.05)$, and LV mass (MSCT: 123 (107-143) g; ECG-MPS: 140 (124-168) g; $p<0.05)$. There were no significant differences in LV EF (MSCT: 64 (54-69)\%; ECG-MPS: 61 (50-66)\%; $p>0.05)$. There was a statistically significant correlation between the values of EDV, ESV, and LV mass $(r=0.81 ; r=0.78 ; r=0.82$, respectively, $p<0.05)$ and a mean correlation of LV EF $(r=0.66 ; p<0.05)$. The Bland-Altman analysis showed that the values of EDV, ESV, and LV mass had statistically significant differences. The consistency limits for the indicators were as follows: EDV, 9-105 ml; ESV, 9-55 ml; LV mass, 51.6-20.7 g. There was a measurement consistency only for EF (consent limits, $16.9-18.4 \% ; p<0.05$ ). Linear regression equations were calculated, which allow determination of exact values for the volume indices and LV mass according ECG-MPS data.

Conclusion. The scintigraphic method versus MSCT yields significantly smaller volumes (EDV and ESV) and higher LV mass. The ECG-MPS values for EDV, ESV, EF, and LV mass have a statistically significant strong correlation with MSCT findings. At the same time, a good consistency of measurements was found only for LV EF.

Keywords: myocardial perfusion scintigraphy; multislice computed tomography; cadmium-zinc-tellurium; left ventricular volume indices; left ventricular mass.

Conflict of interest. The authors declare no conflict of interest.

Acknowledgements. Government task № AAAA-A15-115123110026-3.

For citation: Saushkin V.V., Zavadovskiy K.V. Assessment of accuracy in calculating hemodynamic parameters and left ventricular mass according to ECG-synchronized myocardial perfusion scintigraphic data: comparison with cardiac multislice computed tomography. Journal of Radiology and Nuclear Medicine. 2019; 100 (3): 152-60 (in Russ.). https://doi.org/10.20862/0042-4676-2019-100-3-152-160

Received 13.03.2018 Accepted 26.03.2018

\section{Введение}

Оценка функционального состояния сердца имеет существенное значение в диагностике [1], определении степени тяжести и прогнозе [2, 3] сердечно-сосудистых заболеваний [4]. Так, фракция выброса (ФВ) левого желудочка является основным фактором, определяющим долгосрочную выживаемость пациентов с ИБС. Конечный диастолический объем (КДО) используется как прогностический критерий у пациентов с выраженной сердечной недостаточностью до и после кардиоресинхронизирующей терапии [2]. Повышение конечного систолического объема (КСО) является независимым предиктором сердечной смерти у пациентов после шунтирования коронарных артерий [3]. В свою очередь, точная количественная оценка массы ЛЖ позволяет объективно подтвердить его гипертрофию, что используется для прогнозирования риска внезапной сердечной смерти, сердечной недостаточности и инфаркта 
миокарда, а также определения показаний к реваскуляризации [4].

В настоящее время «золотым стандартом» неинвазивной оценки указанных показателей считается магнитно-резонансная томография (MPT) [5]. При этом многосрезовая компьютерная томография (МСКТ) сердца, имея ряд ограничений (необходимость использования контрастных веществ, лучевая нагрузка), не уступает МРТ по точности определения рассматриваемых показателей [6].

Современным трендом ядерной кардиологии является внедрение в клиническую практику специализированных (кардиологических) гамма-камер с полупроводниковыми кадмий-цинк-теллуровыми детекторами (CZT, cadmium-zinc-telluride) [7, 8]. Они отличаются более высокими показателями чувствительности, пространственного и энергетического разрешения [9].

В то же время в современной зарубежной и особенно отечественной литературе встречается достаточно мало работ, посвященных изучению точности оценки показателей гемодинамики и массы миокарда Лж с использованием CZT-гамма-камер.

Цель нашего исследования - оценить точность вычисления объемов, фракции выброса и массы миокарда ЛЖ, полученных по данным ЭКГ-синхронизированной перфузионной сцинтиграфии миокарда, на гамма-камере с CZT-детекторами, по сравнению с результатами многосрезовой компьютерной томографии сердца.

\section{Материал и методы}

Характеристика пациентов. В исследование были включены 34 пациента (19 мужчин, 15 женщин; средний возраст составил 62 5 лет), которым были выполнены МСКТ-ангиография коронарных артерий (или коронарных шунтов) и перфузионная сцинтиграфия миокарда (ПСМ) с ${ }^{99 m}$ Тс-метоксиизобутилизонитрилом ( ${ }^{99 \mathrm{~m} T \mathrm{C}-М И Б И) ~ п о ~ д в у х д н е в-~}$ ному протоколу «нагрузка-покой». Исследования были проведены на гибридном 64-срезовом ОЭКТ/КТ-томографе Discovery 570c (GE Healthcare, США). Интервал между исследованиями составлял не более 3 дней. Показанием к исследованию у 14 (41\%) пациентов была диагностика ишемической болезни сердца (претестовая вероятность 15-85\%), у 12 (35\%) пациентов - оценка миокардиальной перфузии и состояние коронарных стентов, у 8 (24\%) - оценка состояния аортокоронарных шунтов. Двадцать пациентов (59\%) имели в анамнезе инфаркт миокарда. Критериями исключения из исследования стали: 1) известная аллергия на йодсодержащие контрастные вещества; 2) уровень сывороточного креатинина более 120 мкмоль/л; 3) фибрилляция предсердий; 4) желудочковая аритмия высокой градации по Lown.
Протокол исследования был одобрен комитетом по этике НИИ кардиологии Томского НИМЦ, у пациентов было получено информированное согласие на участие в исследовании.

Сцинтиграфические исследования. Перед выполнением ПСМ у пациентов отменяли прием бета-блокаторов, антагонистов кальция и нитратов за 24 ч до проведения исследования. Коме того, пациентов просили воздержаться от приема производных метилксантина (аминофиллин, кофеин и др.) за 12 ч до фармакологического стресстеста. В качестве стресс-теста использовали внутривенную инфузию аденозином в дозе 140 мг/кг/мин в течение 4 мин. Перфузионную сцинтиграфию миокарда выполняли на пике нагрузочной пробы и в покое. В качестве радиофармпрепарата (РФП) использовали 99mТс-технетрил («Диамед», Россия) в дозе 370-450 МБк. Запись сцинтиграфического изображения выполняли через 1,5 ч после инъекции РФП в ЭКГ-синхронизированном режиме (представительный сердечный цикл был сформирован из 16 кадров). Во время записи пациент находился в положении лежа на спине. Изображения были записаны в течение 10 мин с использованием низкоэнергетического мультипинхол коллиматора (19 проекций, матрица $32 \times 32$ пиксела, размер пиксела 4 мм), с коррекцией аттенюации по данным низкодозной компьютерной томографии. Центр энергетического окна был установлен на фотопик 140 кЭв, ширина энергетического окна составила 20\%. Общая эффективная лучевая нагрузка составила 6-7,3 мЗв. Для дальнейшего анализа были использованы только данные сцинтиграфического исследования, полученные в покое.

Изображения однофотонной эмиссионной КТ (ОЭКТ) были реконструированы с коррекцией аттенюации на специализированной рабочей станции (Xeleris II; GE Healthcare, Haifa, Israel) с использованием алгоритма итеративной реконструкции. При формировании поперечных срезов сердца использовали фильтр Butterworth (частота 0,37, порядок 7). Томографические изображения миокарда, полученные в покое, были обработаны в специализированной программе Corridor 4DM (University of Michigan, Ann Arbor, MI, USA) с использованием срезов по короткой и длинным осям сердца, а также 17-сегментарной полярной карты левого желудочка, нормализованной к 100\%. В полуавтоматическом режиме выполняли оконтуривание миокарда ЛЖ во всех 16 кадрах сердечного цикла, с последующим вычислением КДО, КСО, УО, ФВ и массы миокарда Лж.

МСКТ-коронаро- или шунтрография. Для контрастирования коронарных артерий и/или коронарных шунтов использовали внутривенную инфузию йопамидола (370 мг йода/мл) (Йопамиро, 
ORIGINAL ARTICLES

Bracco, Италия) в объеме 60-110 мл, со скоростью 4-5,5 мл/с. Запись исследования проводили в ретроспективном режиме ЭКГ-синхронизации. Параметры записи были следующие: напряжение на трубке 120 кВ, сила тока 300-600 мА с ЭКГ-модуляцией, скорость вращения трубки 0,4 с, питч: 0,20-0,22 (в зависимости от ЧСС). При проведении исследования лучевая нагрузка составила от 10 до 22 мЗв. Изображения были реконструированы по стандартным протоколам с толщиной срезов 0,625 мм. Из нативных данных были реконструированы 16 фрагментов сердечного цикла (от 0 до 96\% интервала R-R с шагом 8\%).

Компьютерно-томографическая серия была обработана на рабочей станции Advantage Workstations 4.3 (GE Healthcare) с помощью программы Auto ejection fraction программной среды VolumeShare 5. В каждом из 16 кадров автоматически обводили миокард Лж, в ручном режиме корректировали обводку, положение митрального и аортального клапанов. В результате обработки рассчитывали КДО, КСО, УО, ФВ и массу миокарда ЛЖ.

Статистический анализ. Количественные значения представлены в виде медианы (Ме) и межквартильного размаха (Q25-Q75), качественные показатели - в виде абсолютного числа и процентов. Корреляция показателей была оценена с помощью критерия Пирсона. Для оценки значимости различий зависимых переменных применяли критерий Вилкоксона. Согласованность методов исследования оценивали с помощью метода Бланда-Альтмана. Критерием статистической значимости считали значение $p$ менее 0,05. Статистическую обработку проводили в программе Statistica v10 и MedCalc v13.

\section{Результаты}

Клиническая характеристика включенных в исследование пациентов представлена в таблице 1.

По данным перфузионной сцинтиграфии, у 7 (21\%) пациентов миокардиальная перфузия на нагрузке и в покое была нормальная (SSS $<4)$. у $14(41 \%)$ больных было отмечено минимальное (SSS 4-8), у 9 (26\%) - умеренное (SSS 9-13) и у $4(12 \%)$ - выраженное (SSS>13) нарушение
Таблица 1

Клиническая характеристика пациентов

\begin{tabular}{lc}
\hline \multicolumn{1}{c|}{ Параметр } & Число \\
\hline Число мужчин & $19(56 \%)$ \\
Возраст, лет & $62(58-66)$ \\
Класс сердечной недостаточности & \\
по NYHА (I/II/III) & $0 / 30 / 4$ \\
Дислипидемия & $17(50 \%)$ \\
Гипертензия & $22(65 \%)$ \\
Ишемическая болезнь сердца & $30(88 \%)$ \\
Сахарный диабет & $2(6 \%)$ \\
\hline
\end{tabular}

миокардиальной перфузии. У 23 (68\%) пациентов выявлены преходящие, у 11 (32\%) - стабильные дефекты перфузии. Медианные значения SSS coставили 11 (6-15), SRS - 6 (3-9), SDS - 4 (2-6). Объемные показатели и сократительная функция Лж у 28 (82\%) человек имели нормальные значения (ФВ >50\%, КДО < 170 мл), а у 6 (18\%) отмечались дилатация полости ЛЖ и снижение его контрактильной функции (ФВ $<50 \%)$.

Контрастное усиление в левых отделах сердца, по данным МСКТ-исследования, было удовлетворительным у всех пациентов. Значения КДО, КСО, ФВ и массы миокарда левого желудочка, определенные по данным МСКТ и перфузионной сцинтиграфии, представлены в таблице 2.

Медианные значения КДО и КСО, определенные по данным сцинтиграфии, были статистически значимо ниже (разница показателей составила 58 мл (33-70 мл) и 18 мл (7-37 мл) соответственно) по сравнению с результатами МСКТ. Значения фракции выброса ЛЖ, по данным обоих методов, были практически одинаковыми - разница составила 1\% (3-5\%). Достоверных различий по значениям ФВ ЛЖ между методами не выявлено. Масса миокарда, вычисленная с использованием сцинтиграфического метода, была на 26 г (12-31 г) больше, чем по данным МСКТ-исследования.

При проведении корреляционного анализа сильная взаимосвязь была установлена по показателям КДО, КСО и массе миокарда ЛЖ ( $r=0,81$, $r=0,78, r=0,82$ соответственно; $p<0,05)$. Средней

Таблица 2

Показатели гемодинамики и массы миокарда ЛЖ по данным МСКТ и ОЭКТ

\begin{tabular}{|c|c|c|c|c|c|}
\hline Параметр & МСКТ & ОЭКТ & $\begin{array}{c}\text { Wilcoxon } \\
p\end{array}$ & $\begin{array}{l}\text { Correlation } \\
r(p<0,05)\end{array}$ & $\begin{array}{c}\text { Bland-Altman } \\
p\end{array}$ \\
\hline КСО, мл & $72(49-83)$ & $44(32-66)$ & $<0,05$ & 0,78 & $<0,05$ \\
\hline КД0, мл & $168(145-210)$ & $112(94-141)$ & $<0,05$ & 0,81 & $<0,05$ \\
\hline$\Phi \mathrm{B}, \%$ & $64(54-69)$ & $61(50-66)$ & $>0,05$ & 0,66 & $>0,05$ \\
\hline Масса ЛЖ, г & $123(107-143)$ & $140(124-168)$ & $<0,05$ & 0,82 & $<0,05$ \\
\hline
\end{tabular}


силы корреляционная связь выявлена по показателю ФВ ЛЖ ( $r=0,66, p<0,05)$.

При проведении анализа Бланда-Альтмана согласованность методов ОЭКТ и МСКТ в плане оценки объемных показателей и массы миокарда ЛЖ не найдена, полученные значения достоверно отличались между собой (см. табл. 2, рис. 1). Границы согласия методов в измерении КДО Лж 9-105 мл, КСО 9-55 мл. Границы согласия для массы миокарда ЛЖ находились в диапазоне от 51,6 до 20,7 г. В определении ФВ левого желудочка оба метода являются согласованными (средний разброс 0,8\%, граница согласия 16,9-18,4\%, $p<0,05)$ (см. табл. 2). Разница значений имела однонаправленный характер для всех изучаемых показателей.

При проведении регрессионного анализа мы получили уравнения, на основании которых, по данным перфузионной сцинтиграфии миокарда можно определить точные значения КДО, КСО и массы миокарда Лж (рис. 2). В ходе анализа полученные оставшиеся значения для этих трех по- казателей подчинялись закону нормального распределения, что свидетельствует о качестве модели регрессии.

\section{Обсуждение}

Всестороннее изучение функционального состояния сердца, которое включает оценку объемов, фракции выброса и массы миокарда левого желудочка, необходимо для определения тяжести заболевания, выбора метода лечения, в том числе дорогостоящих процедур коронарной реваскуляризации и кардиоресинхронизирующей терапии [10]. Кроме того, вычисление массы миокарда Лж используется для идентификации гипертрофии сердечной мышцы и отслеживания эффекта проведенного лечения [4].

В настоящем исследовании было проведено сопоставление результатов измерения КДО, КСО, ФВ и массы миокарда левого желудочка по данным ОЭКТ на гамма-камере, оснащенной CZT-детекторами, с результатами МСКТ как референсного метода. Показано, что по данным ОЭКТ значе-
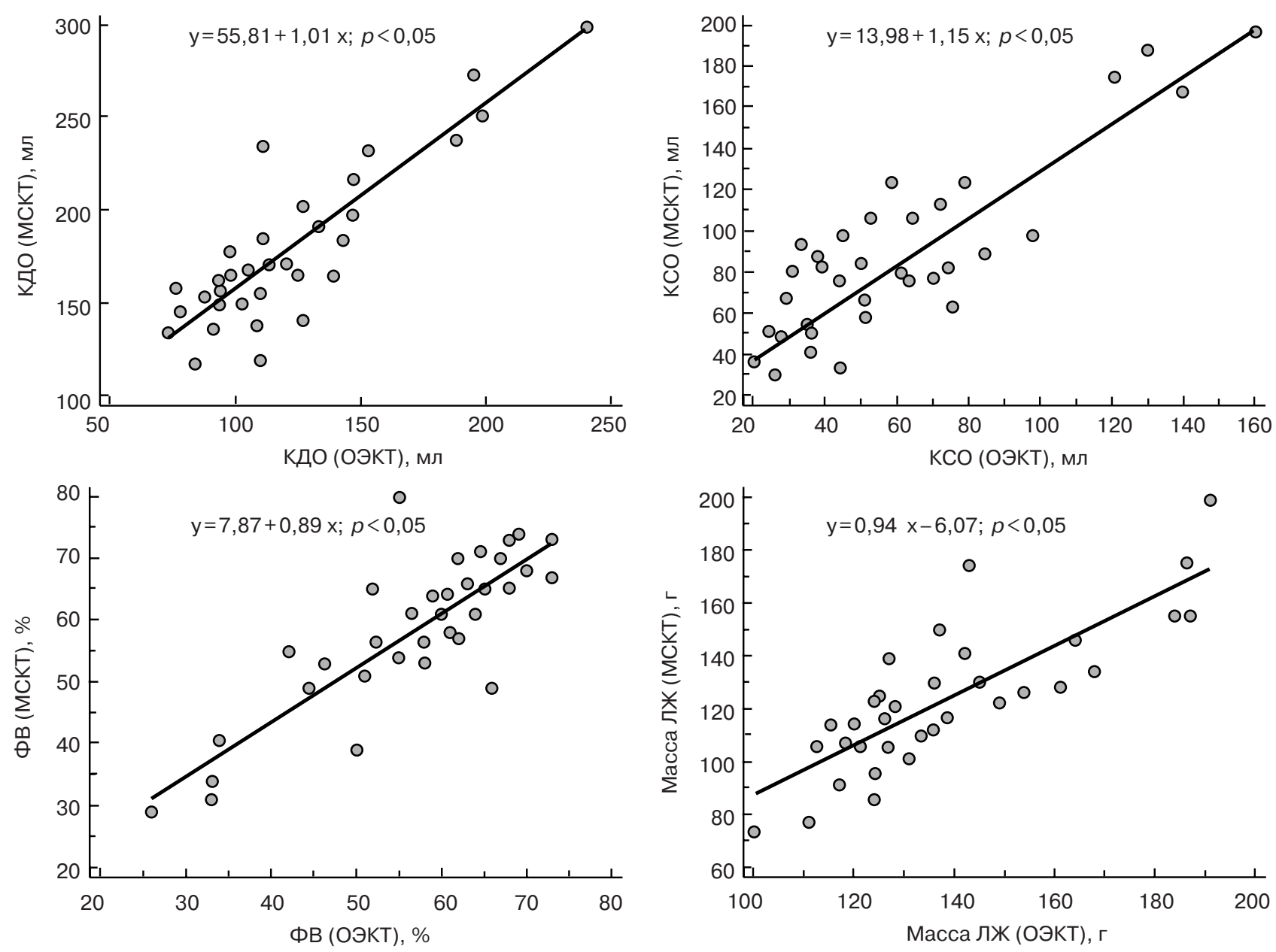

Рис. 1. Результаты линейного регрессионного анализа показателей гемодинамики и массы ЛЖ, вычисленных с помощью ОЭКТ u MCKT 

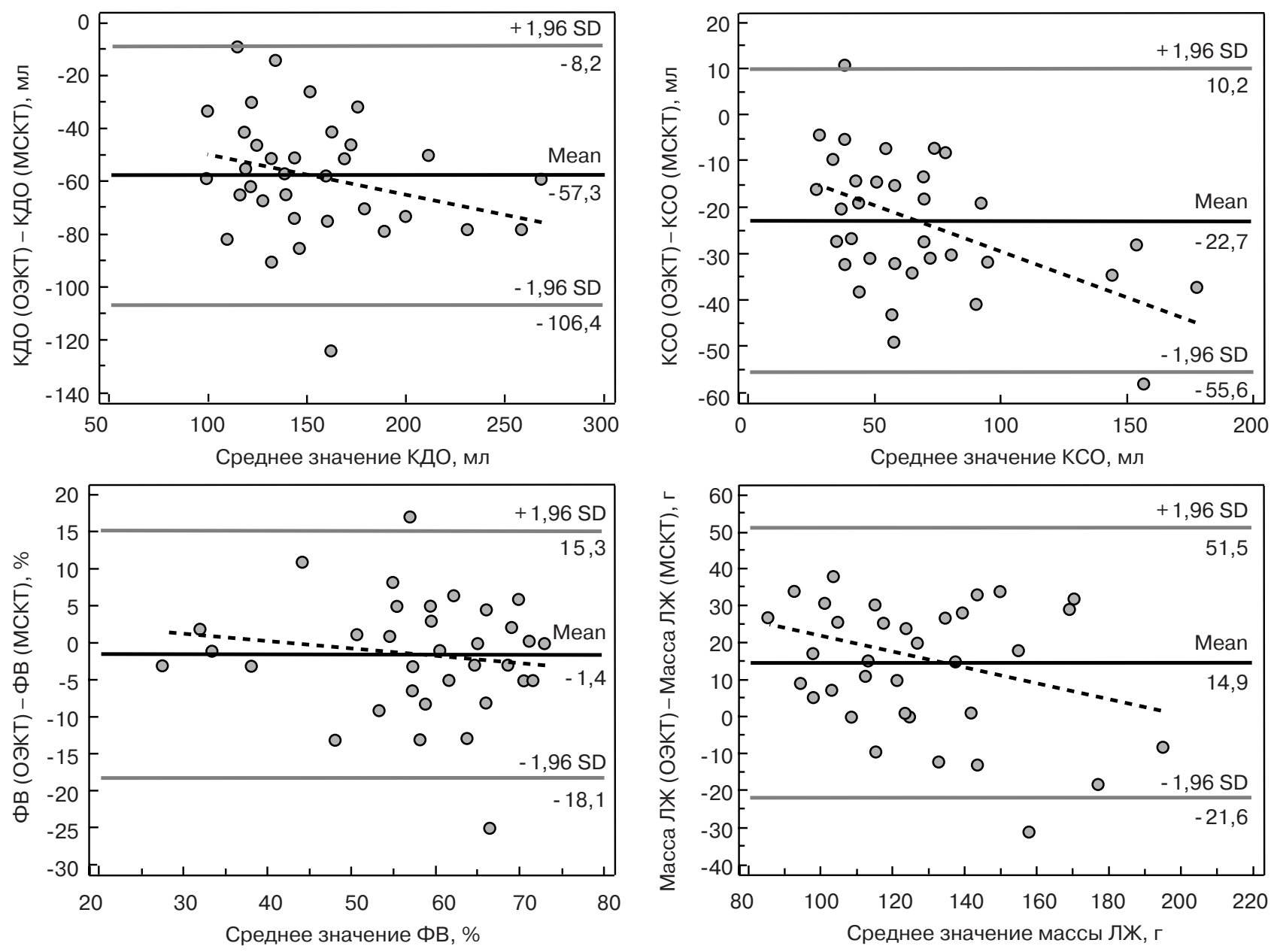

Рис. 2. Результаты анализа Бланда-Альтмана, отражающие согласованность методов ОэКТ и МсКТ в вычислении показателей гемодинамики и массы ЛЖ

ния КДО и КСО Лж были статистически значимо меньше, а масса миокарда - больше, чем по данным МСКТ. Значения ФВ ЛЖ достоверно не различалась. Кроме того, была выявлена статистически значимая корреляция этих показателей, определенных при помощи ОЭКТ и МСКТ.

На сегодняшний день методом выбора для оценки анатомии и функции сердца, а также для количественной оценки массы ЛЖ является МРТ сердца, поскольку она обеспечивает высокое качество изображения, является, по сути, трехмерной и не требует каких-либо математических допущений для расчета объема полостей сердца [2]. Однако высокая стоимость МРТ сердца и ее доступность остаются проблематичными для повседневной клинической практики. Кроме того, МРТ противопоказана для пациентов с имплантированными кардиостимуляторами, а также требует длительного сбора данных и проведения серии эпизодов задержки дыхания, что выполнимо не для всех пациентов кардиологического профиля. Параметры гемодинамики и массу миокарда лж также можно с высокой точностью вычислить при помощи МСКТ сердца [11]. Основными преимуществами данного подхода являются быстрота сканирования (не более 7-10 с на большинстве современных сканеров), а также возможность исследования пациентов с искусственными водителями ритма и кардиоресинхронизирующими устройствами [12]. Данный метод также рассматривается некоторыми авторами как «золотой стандарт» неинвазивного определения основных параметров гемодинамики и сократимости [13]. В то же время существуют факторы, ограничивающие широкое использование МСКТ для указанных целей: необходимость использования йодсодержащих контрастных веществ, а также относительно высокая лучевая нагрузка на пациента (что связано с необходимостью ретроспективной синхронизации записи данных с сигналом ЭКГ) [14].

Еще один томографический метод исследования функционального состояния сердца - ЭКГсинхронизированная перфузионная сцинтиграфия миокарда. На сегодняшний день она пред- 
ставляет собой наиболее распространенный и широко доступный метод определения состояния микроциркуляции миокарда [15-17]. Согласно рекомендациям Европейского общества кардиологов, нагрузочная ОЭКТ миокарда имеет наибольший класс рекомендаций и уровень доказательности у пациентов с промежуточной претестовой вероятностью ИБС [18]. Метод используется как в первичной диагностике ИБС, так и у пациентов с установленным диагнозом для оценки результатов лечения, стратификации риска и прогноза [19]. Кроме миокардиальной перфузии данный подход позволяет оценивать объемы, фракцию выброса, а также массу миокарда ЛЖ. По данным E. Reyes et al., ежегодно в Европе выполняется около 1500 перфузионных сцинтиграфий миокарда на 1 млн населения [19]. Недавнее внедрение в клиническую практику специализированных кардиологических камер на основе детекторов CZT позволило снизить лучевую нагрузку, а также повысить пространственное разрешение и одновременно сократить длительность исследования [8].

В то же время научных работ по сопоставлению результатов определения основных параметров гемодинамики и сократимости сердца, а также массы миокарда ЛЖ по данным ЭКГ-синхронизированной ПСМ на CZT-камерах с результатами МСКТ или МРТ недостаточно, а имеющиеся данные носят противоречивый характер.

Наиболее ранним является исследование H. Cochet et al. [20], в котором было проведено сравнение параметров гемодинамики и сократимости сердца по данным МРТ и сцинтиграфии миокарда (гамма-камера Discovery NM 530c, GE Healthcare) у 60 пациентов как с сохраненной, так и нарушенной глобальной сократимостью ЛЖ. Была выявлена хорошая согласованность результатов оценки фракции выброса ЛЖ, в то время как значения объемов ЛЖ (КДО и КСО) были значимо занижены по данным ОЭКТ-исследования.

A. Giorgetti et al. [8] также выявили недооценку объемов левого желудочка, определенных с использованием ЭКГ-синхронизированной ПСМ, по сравнению с МРТ сердца. Основой для данного вывода послужило исследование 55 пациентов с ИБС, из которых у 29 в анамнезе был инфаркт миокарда (12 человек после шунтирования, 7 после стентирования коронарных артерий).

Сравнению функциональных показателей сердца и массы миокарда ЛЖ по данным ОЭКТ и МСКТ посвящена работа T. Schepis et al. [6]. Авторы выявили высокую степень согласованности и отсутствие статистически значимых различий между методами при изменении ФВ ЛЖ. В то же время по данным МСКТ КДО и КСО были выше, а масса миокарда - ниже, чем по результатам сцинтиграфии.
Несколько другие результаты были получены в недавно опубликованной работе группы авторов под руководством A. Gimelli et al. [7]. В этом исследовании на основании анализа данных 25 пациентов (среди которых 18 перенесли острый инфаркт миокарда) были выявлены отсутствие статистически значимой разницы и высокая степень согласованности всех гемодинамических показателей, а также массы миокарда ЛЖ, измеренных при помощи МРТ и сцинтиграфического метода.

Следует отметить, что во всех рассматриваемых работах значения ФВ имели высокую корреляцию и степень согласованности по данным ОЭКТ и МСКТ, что важно при обследовании пациентов с измененной геометрией Лж (например, при кардиомиопатиях) [21].

Несогласованность объемов, определенных методами ОЭКТ и МСКТ/МРТ, может быть связана с обусловленной наличием дефектов перфузии неточностью оконтуривания эндокардиального и эпикардиального контуров Лж на сцинтиграфических изображениях. В то же время T. Schepis et al. обнаружили сохранение данного феномена даже после исключения из исследования пациентов с нарушениями миокардиальной перфузии [6].

Также известно, что у пациентов с «маленькими» сердцами точность сцинтиграфического определения объемов сердца снижена в связи с артефактами, обусловленными феноменом частичного объемного эффекта [22]. Наиболее значимой причиной более высоких значений объемов ЛЖ по данным МСКТ является включение в измерение выводных отделов ЛЖ (рис. 3). Этот отдел полости ЛЖ не включается в измерение объемов при обработке перфузионных сцинтиграмм [23]. Кроме того, мембранозная часть межжелудочковой перегородки не визуализируется на сцинтиграфических изображениях сердца, в отличие от МСКТ, что приводит к недооценке объемов базальных отделов ЛЖ [24]. Еще одним фактором, определяющим различия объемов, является то, что время конца систолы и конца диастолы может не совпадать при использовании этих методов [6].

Переоценка значений массы миокарда левого желудочка по данным сцинтиграфического исследования связана, вероятнее всего, с феноменом нечеткой визуализации и «размытием» эндокардиального и эпикардиального контуров миокарда. В англоязычных научных публикациях этот феномен носит название «blurring artifact» [25]. Он приводит к некорректно большему объему миокарда, что, в свою очередь, отражается на величине массы миокарда Лж [26]. В нашей работе для нивелирования неточности вычисления КДО, КСО и массы миокарда ЛЖ сцинтиграфическим методом были предложены уравнения линейной регрессии. 

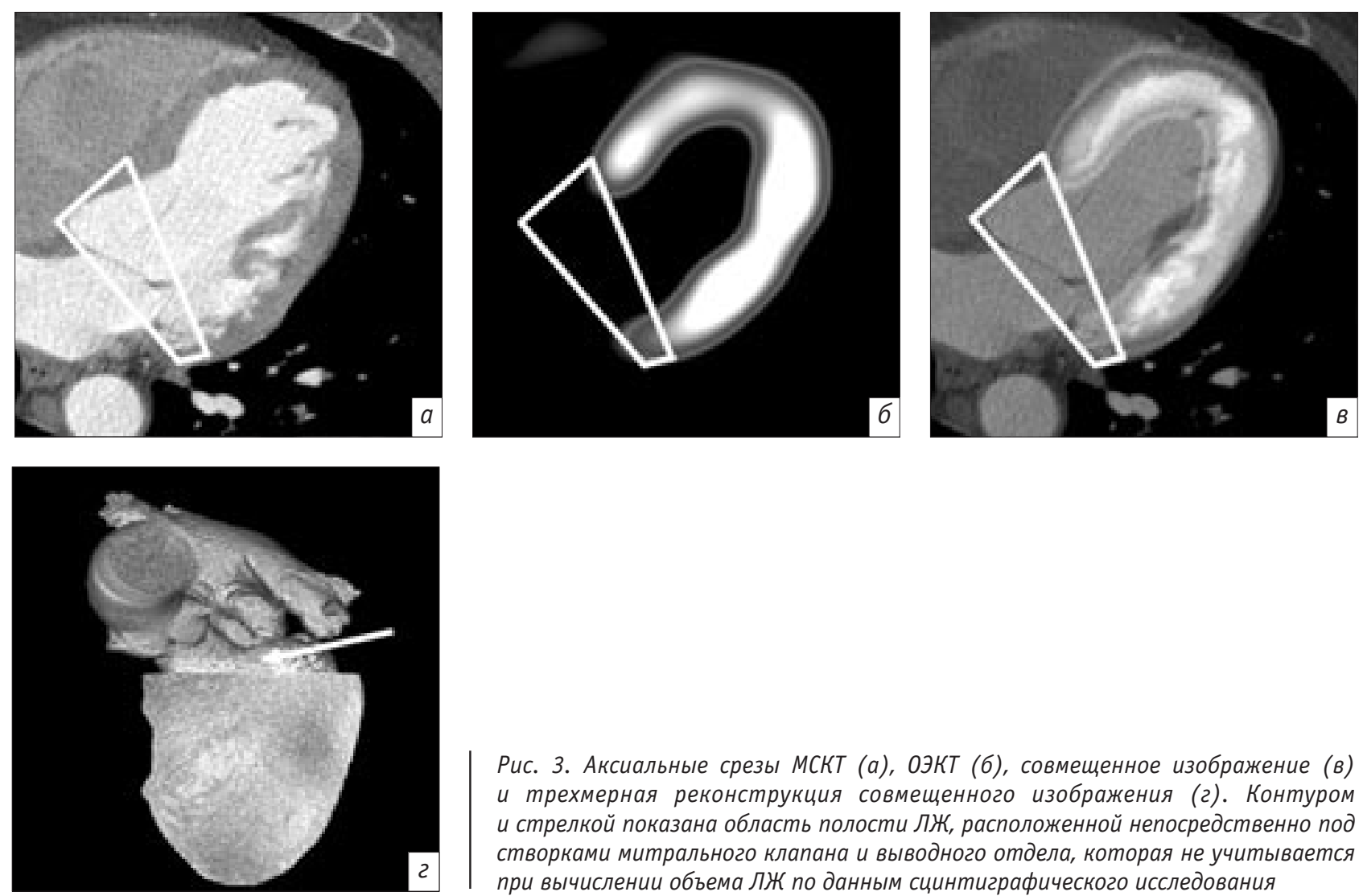

Рис. 3. Аксиальные срезы MСКT (a), ОЭКТ (б), совмещенное изображение (в) и трехмерная реконструкция совмещенного изображения (2). Контуром и стрелкой показана область полости ЛЖ, расположенной непосредственно под створками митрального клапана и выводного отдела, которая не учитывается при вычислении объема ЛЖ по данным сцинтиграфического исследования

\section{Заключение}

Показатели объемов, фракции выброса, а также массы миокарда ЛЖ, вычисленные при помощи ЭКГ-синхронизированной ПСМ на гамма-камере, оснащенной кадмий-цинк-теллуровыми детекторами, имеют статистически значимую сильную корреляцию с результатами МСКТ. При этом хорошая согласованность результатов измерения была выявлена только для фракции выброса ЛЖ. Сцин- тиграфический метод по сравнению с МСКТ показывает значимо меньшие объемы (КДО и КСО) и большие значения массы миокарда левого желудочка. Указанные факторы необходимо принимать во внимание при интерпретации результатов перфузионной сцинтиграфии миокарда, сопоставлении их с данными других лучевых методов и клиническим статусом пациента. Предложенные регрессионные модели могут быть использованы для нивелирования указанной несогласованности.

\section{Литература [References]}

1. Singh P., Bhatt B., Pawar S.U., Kamra A., Shetye S., Ghorpade M. Role of myocardial perfusion study in differentiating ischemic versus nonischemic cardiomyopathy using quantitative parameters. Indian. J. Nucl. Med. 2018; 33 (1): 32-8.

2. Opie L.H., Commerford P.J., Gersh B.J., Pfeffer M.A. Controversies in ventricular remodelling. Lancet. 2006; 367: 356-67.

3. Lima E.G., Carvalho F.P.C., Linhares Filho J.P.P., Pitta F.G., Serrano C.V. Jr. Ischemic left ventricle systolic dysfunction: an evidence-based approach in diagnostic tools and therapeutics. Rev. Assoc. Med. Bras. (1992). 2017; 63 (9): 793-800.

4. Abdi-Ali A., Miller R.J.H., Southern D., Zhang M., Mikami Y., Knudtson M. et al. LV mass independently predicts mortality and need for future revascularization in patients undergoing diagnostic coronary angiography. JACC Cardiovasc. Imaging. 2017. DOI: 10.1016/j.jcmg.2017.04.012

5. Pouleur A.C., de Waroux J.B.P., Pasquet A., Gerber B.L., Ge'rard 0., Allain P. et al. Assessment of left ventricular mass and volumes by three-dimensional echocardiography in patients with or without wall motion abnormalities: comparison against cine magnetic resonance imaging. Heart. 2008; 94: 1050-7.

6. Schepis T., Gaemperli 0., Koepfli P., Valenta I., Strobel K., Brunner A. et al. Comparison of 64-slice CT with gated SPECT for evaluation of left ventricular function. J. Nucl. Med. 2006; 47: 1288-94.

7. Gimelli A., Liga R., Magro S., Novo S., Pedrinelli R., Petronio A.S. et al. Evaluation of left ventricular mass on cadmium-zinctelluride imaging: validation against cardiac magnetic resonance. J. Nucl. Cardiol. 2017. Available at: https://link. springer.com/article/10.1007\%2Fs12350-017-1086-6

8. Giorgetti A., Masci P.G., Marras G., Rustamova Y.K., Gimelli A., Genovesi D. et al. Gated SPECT evaluation of left ventricular function using a CZT camera and a fast low-dose clinical protocol: comparison to cardiac magnetic resonance imaging. Eur. J. Nucl. Med. Mol. Imaging. 2013; 40: 1869-75.

9. Songy B., Lussato D., Guernou M., Queneau M., Geronazzo R. Comparison of myocardial perfusion imaging using thalli- 
um-201 between a new cadmium-zinc-telluride cardiac camera and a conventional SPECT camera. Clin. Nucl. Med. 2011; 36: 776-80.

10. Morishima I., Okumura K., Tsuboi H., Morita Y., Takagi K., Yoshida R. et al. Impact of basal inferolateral scar burden determined by automatic analysis of $99 \mathrm{mTc}$-MIBI myocardial perfusion SPECT on the long-term prognosis of cardiac resynchronization therapy. Europace. 2017; 19: 573-80.

11. Gebhard C., Buechel R.R., Stähli B.E., Gransar H., Achenbach S., Berman D.S. et al. Impact of age and sex on left ventricular function determined by coronary computed tomographic angiography: results from the prospective multicentre CONFIRM study. Eur. Heart. J. Cardiovasc. Imaging. 2017; 18: 990-1000.

12. Al-Mallah M.H., Aljizeeri A., Villines T.C., Srichai M.B., Alsaileek A. Cardiac computed tomography in current cardiology guidelines. J. Cardiovasc. Comput. Tomogr. 2015; 9: 514-23.

13. Asferg C., Usinger L., Kristensen T.S., Abdulla J. Accuracy of multi-slice computed tomography for measurement of left ventricular ejection fraction compared with cardiac magnetic resonance imaging and two-dimensional transthoracic echocardiography: a systematic review and metaanalysis. Eur. J. Radiol. 2012; 81: e757-762.

14. Abbara S., Blanke P., Maroules C.D., Cheezum M., Choi A.D., Han B.K. et al. SCCT guidelines for the performance and acquisition of coronary computed tomographic angiography: a report of the Society of Cardiovascular Computed Tomography Guidelines Committee: Endorsed by the North American Society for Cardiovascular Imaging (NASCI). J. Cardiovasc. Comput. Tomogr. 2016; 10: 435-49.

15. Завадовский К.В., Саушкин В.В., Панькова А.Н., Лишманов Ю.Б. Методические особенности выполнения, обработки результатов и интерпретации данных радионуклидной равновесной томовентрикулографии. Радиология практика. 2011; 6: 75-83.

[Zavadovskiy K.V., Saushkin V.V., Pan'kova A.N., Lishmanov Yu.B. Methodological features of gated blood pool spect data acquisition, imaging processing and results interpretation. Radiologiya - Praktika (Radiology - Practice). 2011; 6: 75-83 (in Russ.).]

16. Аншелес А.А. Особенности интерпретации перфузионной однофотонной эмиссионной компьютерной томографии миокарда с компьютерно-томографической коррекцией поглощения. Вестник рентгенологии и радиологии. 2014; 95 (2): 5-20.

[Ansheles A.A. Specific features of interpretation of myocardial perfusion single-photon emission computed tomography with computed tomographic absorption correction. Journal of Radiology and Nuclear Medicine. 2014; 95 (2): 5-20 (in Russ.).]
17. Сергиенко В.Б., Аншелес А.А. Радионуклидная диагностика в кардиологии. В кн.: Чазов Е.И. (ред.) Руководство по кардиологии. В 4-х т. М.: Практика; 2014; 2: 571-612. [Sergienko V.B., Ansheles A.A. Radionuclide diagnostics in cardiology. In: Chazov E.I. (Ed.) The guide to cardiology. In 4th Vol. Moscow: Praktika; 2014; 2: 571-612 (in Russ.).]

18. Montalescot G., Sechtem U., Achenbach S., Andreotti F., Arden C., Budaj A. et al. 2013 ESC guidelines on the management of stable coronary artery disease: the task force on the management of stable coronary artery disease of the European Society of Cardiology. Eur. Heart J. 2013; 34: 2949-3003.

19. Reyes E., Wiener S., Underwood S.R., European Council of Nuclear Cardiology. Myocardial perfusion scintigraphy in Europe 2007: a survey of the European Council of Nuclear Cardiology. Eur. J. Nucl. Med. Mol. Imaging. 2012; 39: 160-4.

20. Cochet H., Bullier E., Gerbaud E., Durieux M., Godbert Y., Lederlin M. et al. Absolute quantification of left ventricular global and regional function at nuclear MPI using ultrafast CZT SPECT: initial validation versus cardiac MR. J. Nucl. Med. 2013; 54: 556-63.

21. Bax J.J., Lamb H., Dibbets P., Pelikan H., Boersma E., Viergever E.P. et al. Comparison of gated single-photon emission computed tomography with magnetic resonance imaging for evaluation of left ventricular function in ischemic cardiomyopathy. Am. J. Cardiol. 2000; 86: 1299-305.

22. Nakajima K., Okuda K., Nyström K., Richter J., Minarik D., Wakabayashi $\mathrm{H}$. et al. Improved quantification of small hearts for gated myocardial perfusion imaging. Eur. J. Nucl. Med. Mol. Imaging. 2013; 40: 1163-70.

23. Kaufmann P.A. Measurement of left ventricular volumes and function using 0-15-labeled carbon monoxide gated PET. J. Nucl. Cardiol. 2005; 12: 620-1.

24. Kondo C., Watanabe E., Momose M., Fukushima K., Abe K., Hagiwara N., Sakai S. In vivo validation of gated myocardial SPECT imaging for quantification of small hearts: comparison with cardiac MRI. EJNMMI Res. 2016; 6 (1): 9.

25. Suzuki Y., Matsumoto N., Nakano Y., Miki T., Igarashi Y., Sato Y. et al. Motion-frozen myocardial perfusion database obtained from Japanese population shows same tendency of count distribution of the American datasets. J. Nucl. Cardiol. 2009; 16: 662.

26. Завадовский К.В., Мишкина А.И., Мочула А.В., Лишманов Ю.Б. Методика устранения артефактов движения сердца при выполнении перфузионной сцинтиграфии миокарда. REJR. 2017; 7 (2): 56-64. DOI: 10.21569/ 2222-7415-2017-7-2-56-64

[Zavadovskiy K.V., Mishkina A.I., Mochula A.V., Lishmanov Yu.B. The method for correction of motion artefacts to improve myocardial perfusion imaging. REJR. 2017; 7 (2): 56-64 (in Russ.). DOI: 10.21569/2222-7415-2017-7-2-56-64]

\section{Сведения об авторах | Information about the autors}

Саушкин Виктор Вячеславович*, к. м. Н., ст. науч. сотр., Научно-исследовательский институт кардиологии ФГБНУ «Томский национальный исследовательский медицинский центр Российской академии наук»; orcid.org/0000-0001-5564-3802

E-mail: vitversus@gmail.com

Завадовский Константин Валерьевич, д. М. Н., руководитель лаборатории радионуклидных методов исследования, Научноисследовательский институт кардиологии ФГБНУ «Томский национальный исследовательский медицинский центр Российской академии наук»; orcid.org/0000-0002-1513-8614
Viktor V. Saushkin*, Cand. Med. Sc., Senior Researcher, Cardiology Research Institute, Tomsk National Research Medical Center of the Russian Academy of Sciences; orcid.org/0000-0001-5564-3802

E-mail:vitversus@gmail.com

Konstantin V. Zavadovskiy, Dr. Med. Sc., Head of Laboratory of Radionuclide Methods of Study, Cardiology Research Institute, Tomsk National Research Medical Center of the Russian Academy of Sciences; orcid.org/0000-0002-1513-8614 